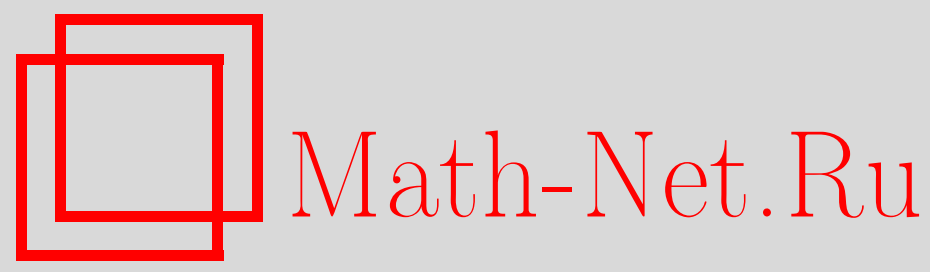

В. П. Чистяков, Асимптотическая нормальность числа значений $m$-зависимых случайных величин, появившихся заданное число раз, Дискрет. матем., 2011, том 23, выпуск $2,41-52$

DOI: https://doi.org/10.4213/dm1139

Использование Общероссийского математического портала Math-Net.Ru подразумевает, что вы прочитали и согласны с пользовательским соглашением http://www.mathnet.ru/rus/agreement

Параметры загрузки:

IP: 54.198 .67 .100

26 апреля 2023 г., $15: 20: 37$ 


\title{
Асимптотическая нормальность числа значений m-зависимых случайных величин, появившихся заданное число раз
}

\author{
() 2011 г. . П. Чистяков
}

\begin{abstract}
Для стационарной последовательности случайных величин $x_{1}, x_{2}, \ldots, x_{t}, \ldots$, каждая из которых принимает значения из множества $\mathcal{N}=\{1, \ldots, N\}$, изучаются случайные величины $\mu_{r}=\mu_{r}(n, N)$, равные числу значений из множества $\mathcal{N}$, появившихся $r$ раз в первых $n$ членах последовательности. Получены асимптотические формулы моментов $\mu_{r}(n, N)$ и достаточные условия асимптотической нормальности этой величины, когда $n, N \rightarrow \infty$ и последовательность является последовательностью $m$-зависимых величин общего вида, а параметры распределения последовательности изменяются в так называемой центральной области. Случайные величины $\mu_{r}(n, N)$ при $n, N \rightarrow \infty$ в левой промежуточной области изучались ранее.
\end{abstract}

\section{1. Введение}

Пусть

$$
x_{1}, x_{2}, \ldots, x_{t}, \ldots
$$

- стационарная последовательность случайных величин, каждая из которых может принимать значения из множества $\mathcal{N}=\{1, \ldots, N\}$. Обозначим $\mu_{r}=\mu_{r}(n, N)$ число значений из множества $\mathcal{N}$, появившихся $r$ раз в первых $n$ членах последовательности (1). Асимптотические формулы для $\mathbf{E} \mu_{r}, \mathbf{E} \mu_{r}^{[2]}, \mathbf{D} \mu_{r}$ и асимптотическая нормальность $\mu_{r}$ при $n, N \rightarrow \infty$ рассматривались многими авторами (см., например, [1-3]) в случае, когда случайные величины (1) независимы или образованы $s$-цепочками независимых величин.

В данной работе получены асимптотические формулы моментов $\mu_{r}(n, N)$ и достаточные условия асимптотической нормальности этой величины, когда $n, N \rightarrow \infty$, последовательность (1) является последовательностью $m$-зависимых величин общего вида, а параметры распределения последовательности (1) изменяются по терминологии [1] в центральной области. В $[4,5]$ исследовались величины $\mu_{r}(n, N)$ при $n, N \rightarrow \infty$ в левой промежуточной области.

\section{2. Постановка задачи}

Будем предполагать, что последовательность (1) является последовательностью $m$-зависимых случайных величин: случайные векторы $\left(x_{s-u}, x_{s-u+1}, \ldots, x_{s}\right),\left(x_{t}, x_{t+1}, \ldots, x_{t+v}\right)$ 
независимы при любых $u, v, s, t$, у которых $t-s>m$. Введем обозначения

$$
a_{i}=N \mathbf{P}\left\{x_{t}=i\right\}, \quad b_{i j}(k)=N^{2} \mathbf{P}\left\{x_{t}=i, x_{t+k}=j\right\}, \quad i, j \in \mathbf{N}, \quad t=1,2, \ldots
$$

Отметим, что

$$
\sum_{i=1}^{N} a_{i}=N, \quad \sum_{i, j=1}^{N} b_{i j}(k)=N^{2}, \quad k=1,2, \ldots, \quad b_{i j}(k)=a_{i} a_{j}, \quad k \geqslant m+1 .
$$

Случайную величину $\mu_{r}(n, N)$ можно представить в виде

$$
\mu_{r}=\mu_{r}(n, N)=\sum_{i=1}^{N} \sum_{1 \leqslant h_{1}<\ldots<h_{r} \leqslant n} \chi\left(L_{i}(h) \cap\left(x_{t} \neq i, 1 \leqslant t \leqslant n, t \notin h\right)\right),
$$

где $\chi(A)$ - индикатор события $A, h=\left(h_{1}, \ldots, h_{r}\right)$,

$$
L_{i}(h)=\bigcap_{t \in h}\left(x_{t}=i\right) .
$$

Асимптотические формулы моментов случайных величин $\mu_{r}(n, N)$ получены в схеме серий, когда $n, N \rightarrow \infty$

$$
\frac{n}{N}=\alpha_{N}, \quad 0<\underline{\alpha} \leqslant \alpha_{N} \leqslant \bar{\alpha}<\infty
$$

и совместные распределения случайных величин (1) меняются так, что при любых $t_{1}<\ldots<t_{l}$ и любых $i_{1}, \ldots, i_{l} \in \mathbf{N}, l=1,2 \ldots$

$$
0<\underline{a} \leqslant a_{i} \leqslant \bar{a}<\infty, \quad N^{l} \mathbf{P}\left\{x_{t_{1}}=i_{1}, \ldots, x_{t_{l}}=i_{l}\right\} \leqslant c^{l},
$$

где $\underline{a}, \bar{a}, c-$ некоторые постоянные.

\section{3. Математическое ожидание}

Слагаемые в правой части равенства (3) представим в виде

$$
\chi\left(L_{i}(h) \cap \bigcap_{t \in h}\left(x_{t} \neq i\right)\right)=\chi\left(L_{i}(h)\right)-\chi\left(L_{i}(h) \cap \bigcup_{t \in \bar{h}}\left(x_{t}=i\right)\right),
$$

где $\bar{h}=\{1,2, \ldots, n\} \backslash h$. Отсюда, используя равенство

$$
\chi\left(L_{i}(h) \cap \bigcup_{t \in \bar{h}}\left(x_{t}=i\right)\right)=\sum_{l=1}^{n-r}(-1)^{l+1} \sum_{\bar{t}(l) \in T_{h}(l)} \chi\left(L_{i}(h) \cap\left(x_{t_{1}}=\ldots=x_{t_{l}}=i\right)\right)
$$

и равенство

$$
\begin{aligned}
& \sum_{1 \leqslant h_{1}<\ldots<h_{r} \leqslant n} \sum_{t(l) \in T_{h}(l)} \chi\left(L_{i}(h) \cap\left(x_{t_{1}}=\ldots=x_{t_{l}}=i\right)\right) \\
&=\left(\begin{array}{c}
l+r \\
r
\end{array}\right) \sum_{t(l+r) \in T(l+r)} \chi\left(x_{t_{1}}=\ldots=x_{t_{l}}=i\right),
\end{aligned}
$$


где

$$
\begin{aligned}
t(l) & =\left(t_{1}, \ldots, t_{l}\right), \\
t(l+r) & =\left(t_{1}, \ldots, t_{l+r}\right), \\
T(l+r) & =\left\{t(l+r): 1 \leqslant t_{1}<\ldots<t_{l+r} \leqslant n\right\}, \\
T_{h}(l) & =\left\{t(l): 1 \leqslant t_{1}<\ldots<t_{l} \leqslant n ; t_{1}, \ldots, t_{l} \notin h\right\},
\end{aligned}
$$

получим равенство

$$
\mu_{r}=\sum_{l=r}^{\varphi-1} \sum_{i=1}^{N}(-1)^{l+r}\left(\begin{array}{l}
l \\
r
\end{array}\right) \sum_{t(l) \in T(l)} \chi\left(x_{t_{1}}=\ldots=x_{t_{l}}=i\right)+\Delta_{\varphi},
$$

где $1<\varphi<n-r$,

$$
\left|\Delta_{\varphi}\right| \leqslant \sum_{i=1}^{N}\left(\begin{array}{l}
\varphi \\
r
\end{array}\right) \sum_{t(\varphi) \in T(\varphi)} \chi\left(x_{t_{1}}=\ldots=x_{t_{\varphi}}=i\right) .
$$

Отсюда

$$
\begin{aligned}
\mathbf{E} \mu_{r} & =\sum_{l=r}^{\varphi-1} \sum_{i=1}^{N}(-1)^{l+r}\left(\begin{array}{l}
l \\
r
\end{array}\right) \sum_{\bar{t}(l) \in T(l)} \mathbf{P}\left(x_{t_{1}}=\ldots=x_{t_{l}}=i\right)+\mathbf{E} \Delta_{\varphi}, \\
\left|\mathbf{E} \Delta_{\varphi}\right| & \leqslant \sum_{i=1}^{N}\left(\begin{array}{l}
\varphi \\
r
\end{array}\right) \sum_{t(\varphi) \in T(\varphi)} \mathbf{P}\left(x_{t_{1}}=\ldots=x_{t_{\varphi}}=i\right) .
\end{aligned}
$$

Определим $\varphi$ равенством

$$
\varphi=\left[\frac{(1+\delta) \ln N}{\ln \ln N}\right]
$$

Положим $\tau(t)=(t, t+1, \ldots, t+m)$. Множества $T(l)$ представим в виде объединения трех непересекающихся множеств:

$$
\begin{aligned}
T(l) & =T_{1}(l) \cup T_{2}(l) \cup T_{3}(l), \\
T_{1}(l) & =\left\{t(l): \tau\left(t_{u}\right) \cap \tau\left(t_{v}\right)=\varnothing, u, v=1, \ldots, l, u \neq v\right\}, \\
T_{2}(l) & =\left\{t(l): \text { среди } \tau\left(t_{1}\right), \ldots, \tau\left(t_{l}\right) \text { ровно одна пара множеств пересекается }\right\}, \\
T_{3}(l) & =T(l) \backslash\left(T_{1}(l) \cap T_{2}(l)\right) .
\end{aligned}
$$

Сумму по $\bar{t}(l) \in T(l)$ в равенстве (9) разобьем на три суммы, соответствующие разбиениям (12).

Положим

$$
\begin{aligned}
A_{k}(l) & =\sum_{i=1}^{N} \sum_{t(l) \in T_{k}(l)} \mathbf{P}\left(x_{t_{1}}=\ldots=x_{t_{l}}=i\right), \\
E_{k} & =\sum_{l=r}^{\varphi-1}(-1)^{l+r} A_{k}(l)\left(\begin{array}{l}
l \\
r
\end{array}\right), \quad k=1,2, \ldots
\end{aligned}
$$


Отсюда и из (9), (10) получим, что

$$
\mathbf{E} \mu_{r}=E_{1}+E_{2}+E_{3}+\mathbf{E} \Delta_{\varphi},
$$

где

$$
\left|\Delta_{\varphi}\right| \leqslant \Delta_{1}+\Delta_{2}+\Delta_{3}, \quad \Delta_{k}=\left(\begin{array}{l}
\varphi \\
r
\end{array}\right) A_{k}(\varphi), \quad k=1,2,3 .
$$

В схеме серий (4), (5) найдем асимптотические формулы для $A_{k}(l), E_{k}$.

По лемме 5 статьи [2] (в лемме 5 нужно $s$ заменить на $m+1$ ):

$$
\left|T_{1}(l)\right|=\frac{n^{l}}{l !}-\frac{n^{l-1}}{(l-2) !} \frac{2 m+1}{2}+O\left(\frac{l^{3} n^{l-2}}{(l-2) !}\right), \quad l \geqslant 2 ;
$$

кроме того,

$$
\left|T_{1}(0)\right|=1, \quad\left|T_{1}(1)\right|=n .
$$

При $t(l) \in T_{1}(l)$ величины $x_{t_{1}}, \ldots, x_{t_{l}}$ независимы и, следовательно,

$$
\mathbf{P}\left(x_{t_{1}}=\ldots=x_{t_{l}}=i\right)=\frac{a_{i}^{l}}{N^{l}}, \quad i=1, \ldots, N .
$$

Отсюда, используя (15), получим, что

$$
\begin{aligned}
A_{1}(l) & =\sum_{i=1}^{N}\left|T_{1}(l)\right| \frac{a_{i}^{l}}{N^{l}} \\
& =\sum_{i=1}^{N}\left[\frac{\left(\alpha_{N} a_{i}\right)^{l}}{l !}-\frac{\left(\alpha_{N} a_{i}\right)^{2}}{\alpha_{N} N} \frac{2 m+1}{2} \alpha_{N} \frac{\left(\alpha_{N} a_{i}\right)^{l-2}}{(l-2) !}+O\left(\frac{1}{N^{2}} \frac{l^{3}}{(l-2) !}\right)\right] ;
\end{aligned}
$$

кроме того,

$$
A_{1}(\varphi) \leqslant C N \frac{c_{0}^{\varphi}}{\varphi !}<\frac{C}{N^{\delta_{0}}}, \quad \Delta_{1}<\frac{C \varphi^{2}}{N^{\delta_{0}}}<\frac{C}{N^{\delta}}, \quad 0<\delta<1,
$$

где $C>0, c_{0}>0,0<\delta<1,0<\delta_{0}<1-$ некоторые постоянные.

Подставим выражение для $A_{1}(l)$ во второе равенство (13) при $k=1$. Заменив верхний предел суммирования $\varphi-1$ на $\infty$, с учетом оценки остатка ряда с $l \geqslant \varphi$, получим равенство

$$
E_{1}=\sum_{i=1}^{N} p_{r, i}\left(1-\frac{2 m+1}{2 N \alpha_{N}}\left(r(r-1)-2 r\left(\alpha_{N} a_{i}\right)+\left(\alpha_{N} a_{i}\right)^{2}\right)\right)+O\left(\frac{1}{N^{\delta}}\right),
$$

где $0<\delta<1$,

$$
p_{r, i}=\frac{\alpha_{N}}{r !} e^{-\alpha_{N} a_{i}}
$$

Оценим $A_{2}(l)$. Для $l \geqslant 2$ определим множества

$$
T_{2}^{u}(l, v, n)=\left\{t(l): t(l) \in T(l), t_{v+1}-t_{v}=u, t_{k+1}-t_{k} \geqslant m+1 \text { при } k \neq v\right\} .
$$


Используя равенство

$$
\mathbf{P}\left(x_{t_{1}}=\ldots=x_{t_{l}}=i\right)=\frac{b_{i i}(u)}{N^{2}} \frac{a_{i}^{l-2}}{N^{l-2}}, \quad t(l) \in T_{2}^{u}(l, v, n),
$$

получим, что

$$
A_{2}(l)=\sum_{u=1}^{m} \sum_{v=1}^{l-1}\left|T_{2}^{(u)}(l, v, n)\right| \sum_{i=1}^{N} \frac{b_{i i}(u) a_{i}^{l-2}}{N^{l}} .
$$

Отсюда и из равенства

$$
\sum_{v=1}^{l-1}\left|T_{2}^{(u)}(l, v, n)\right|=\frac{n^{l-1}}{(l-2) !}+O\left(\frac{l^{3} n^{l-2}}{(l-2) !}\right)
$$

(см. равенство (31) в [2]) следует, что

$$
A_{2}(l)=\frac{\alpha_{N}}{N} \sum_{i=1}^{N} \frac{\left(\alpha_{N} a_{i}\right)^{(l-2)}}{(l-2) !} B_{i i}+O\left(\frac{1}{N} \frac{l^{3}}{(l-2) !}\right),
$$

где

$$
B_{i j}=\sum_{u=1}^{m} b_{i j}(u)
$$

Используя равенства (13), (21), получим, что

$$
E_{2}=\frac{\alpha_{N}}{r ! N} \sum_{i=1}^{N}\left(\alpha_{N} a_{i}\right)^{r-2} B_{i i} \sum_{l=0}^{\infty}(-1)^{l}(r(r-1)+2 r l+l(l-1)) \frac{\left(\alpha_{N} a_{i}\right)^{l}}{l !}+O\left(\frac{1}{N^{\delta}}\right) .
$$

Отсюда следует, что

$$
\mathbf{E}_{2}=\frac{\alpha_{N}}{N} \sum_{i=1}^{N} B_{i i}\left(p_{r-2, i}-2 p_{r-1, i}+p_{r, i}\right)+O\left(\frac{1}{N^{\delta}}\right), \quad 0<\delta<1,
$$

где $p_{r, i}$ определены равенством (19). Из равенств (14) и (21) следует, что

$$
\begin{aligned}
& A_{2}(\varphi)<\frac{c_{0}^{\varphi}}{(\varphi) !}<\frac{1}{N^{1+\delta_{0}}}, \\
& \Delta_{2}<C \varphi^{r} \frac{c_{0}^{\varphi}}{(\varphi) !}<\frac{1}{N^{1+\delta_{0}}}, \quad 0<\delta_{0}<1, \quad 0<\delta_{1}<1 .
\end{aligned}
$$

Используя равенство

$$
\left|T_{3}(l)\right|=O\left(\frac{n^{l-2}}{(l-2) !}\right)
$$

получим оценки

$$
E_{3}=O\left(\frac{1}{N^{\delta}}\right), \quad \Delta_{3}=O\left(\frac{1}{N^{2+\delta}}\right)
$$


Собирая полученные асимптотические формулы и оценки, получим следующую теорему 1 .

Воспользуемся обозначениями

$$
\begin{array}{rlrl}
P_{r, i} & =\frac{\left(\alpha_{N} a_{i}\right)^{r}}{r !} e^{-\alpha_{N} a_{i}}, & M_{r} & =\sum_{i=1}^{N} p_{r, i}, \\
p_{r} & =\sum_{i=1}^{N}\left(\frac{B_{i i}}{m a_{i}^{2}}-1\right) p_{r, i}, & Q_{r_{1}, r_{2}} & =\sum_{i, j=1}^{N}\left(\frac{B_{i j}}{m a_{i} a_{j}}-1\right) p_{r_{1}, i} p_{r_{2}, j}, \\
B_{i j} & =\sum_{u=1}^{m} b_{i j}(u),
\end{array}
$$

которые использованы при формулировке теоремы 1 и далее при выводе асимптотических формул для $\mathbf{E} \mu_{r}\left(\mu_{r}-1\right), \mathbf{D} \mu_{r}$.

Теорема 1. При $n, N \rightarrow \infty$ в схеме серий (4), (5)

$$
\begin{aligned}
\mathbf{E} \mu_{r}=M_{r} & -\frac{1}{2 \alpha_{N} N}\left(r(r-1) M_{r}-2 r(r+1) M_{r+1}+(r+1)(r+2) M_{r+2}\right) \\
& +\frac{m}{\alpha_{N} N}\left(r(r-1) p_{r}-2 r(r+1) p_{r+1}+(r+1)(r+2) p_{r+2}\right) \\
& +O\left(\frac{1}{N^{\delta}}\right),
\end{aligned}
$$

где $0<\delta<1$.

\section{4. Дисперсия}

Получим сначала асимптотическую формулу для $\mathbf{E} \mu_{r}\left(\mu_{r}-1\right)$. Используя (3), представим $\mu_{r}^{[2]}=\mu_{r}\left(\mu_{r}-1\right)$ в виде

$$
\mu_{r}^{[2]}=\sum_{(i, h) \neq(j, g)} \chi\left(L_{i}(h) \cap L_{j}(g) \cap \bigcup_{t \in \bar{h}}\left(x_{t} \neq i\right) \cap \bigcup_{t \in \bar{g}}\left(x_{t} \neq j\right)\right),
$$

где

$$
\begin{array}{ll}
h=\left(h_{1}, \ldots, h_{r}\right), \quad & g=\left(g_{1}, \ldots, g_{r}\right), \\
\bar{h}=\{1, \ldots, n\} \backslash h, & \bar{g}=\{1, \ldots, n\} \backslash g,
\end{array}
$$

суммирование проводится по всем $i, j \in\{1, \ldots, N\}$ и всем подмножествам $h, g$ множества $\{1,2, \ldots, n\}$.

Отсюда следует, что

$$
\mathbf{E} \mu_{r}^{[2]}=\sum_{(i, h) \neq(j, g)} p(i, j, h, g)
$$

где

$$
p(i, j, h, g)=p\left(L_{i}(h) \cap L_{j}(g) \cap \bigcap_{t \in \bar{h}}\left(x_{t} \neq i\right) \cap \bigcap_{t \in \bar{g}}\left(x_{t} \neq j\right)\right) .
$$


Нетрудно проверить, что

$$
\begin{aligned}
& p(i, i, h, g)=0, \quad h \neq g, \\
& p(i, j, h, h)=0, \quad i \neq j, \quad h \cap g \neq \varnothing .
\end{aligned}
$$

Таким образом, в сумме (27) остаются слагаемые, у которых $i \neq j$ и $h \cap g=\varnothing$. В этом случае

$$
p(i, j, h, g)=p\left(L_{i}(h) \cap L_{j}(g)\right)-p\left(L_{i}(h) \cap L_{j}(g) \cap\left(\bigcup_{t \in \overline{h \cup g}}\left(\left(x_{t}=i\right) \cap\left(x_{t}=j\right)\right)\right)\right)
$$

и

$$
p(i, j, h, g)=p\left(L_{i}(h) \cap L_{j}(g)\right)-\sum_{l=1}^{n-2 r}(-1)^{l+1} z \sum_{t(l) \in T_{h \cup g}(l)} p_{i j}(h, g, f),
$$

где

$$
\begin{aligned}
f(l) & =\left(f_{1}, \ldots, f_{l}\right), \\
T_{h \cup g}(l) & =\left\{f(l): 1 \leqslant f_{1}<\ldots<f_{l} \leqslant n ; f_{1}, \ldots, f_{l} \notin h \cup g\right\}, \\
p_{i j}(h, g, f(l)) & =p\left(L_{i}(h) \cap L_{j}(g) \cap \bigcap_{t \in f(l)}\left(\left(x_{t}=i\right) \cup\left(x_{t}=j\right)\right)\right) .
\end{aligned}
$$

Используя равенства (27), (28), (29), получим равенство

$$
\mathbf{E} \mu_{r}^{[2]}=\sum_{i \neq j} \sum_{l=0}^{\varphi-1}(-1)^{l} z \sum_{t(l+2 r) \in T(l+2 r)} \sum_{h, g, f} p_{i j}(h, g, f)+\Delta_{\varphi},
$$

где

$$
\begin{gathered}
t(l+2 r)=\left(t_{1}, \ldots, t_{l+2 r}\right), \quad T(l+2 r)=\left\{t(l+2 r): 1 \leqslant i_{1}<\ldots<t_{l+2 r} \leqslant n\right\}, \\
\left|\Delta_{\varphi}\right| \leqslant \sum_{i \neq j} \sum_{t(\varphi) \in T(\varphi)} \sum_{h, g, f} p_{i j}(h, g, f)
\end{gathered}
$$

и суммирование по $h, g, f$ проводится по всем разбиениям множества

$$
t(l+2 r)=h \cup g \cup f .
$$

Так же, как и при выводе асимптотической формулы для $\mathbf{E} \mu_{r}$, сумму в (30) по $t(l+2 r) \in T(l+2 r)$ мы разобьем на три суммы, соответствующие разбиениям (12) с заменой $l$ на $l+2 r$.

Положим

$$
D_{k}(l)=\sum_{i \neq j} \sum_{t(l+2 r) \in T_{k}(l+2 r)} \sum_{h, g, f} p_{i j}(h, g, f), \quad k=1,2,3,
$$

где $T_{k}(l+2 r)$ определяется равенствами (12) с заменой $l$ на $l+2 r$. Тогда

$$
\mathbf{E} \mu_{r}^{[2]}=\sum_{l=0}^{\varphi-1}(-1)^{l}\left(D_{1}(l)+D_{2}(l)+D_{3}(l)\right)+\Delta_{\varphi},
$$


где $\left|\Delta_{\varphi}\right|<\Delta_{1}+\Delta_{2}+\Delta_{3}$,

$$
\Delta_{k}=\sum_{i \neq j} \sum_{t(\varphi) \in T(\varphi)} p_{i j}(h, g, f), \quad k=1,2,3 .
$$

При $t(l+2 r) \in T_{1}(l+2 r)$ события, определяемые вектором $\left(x_{t_{1}}, \ldots, x_{t_{l+2 r}}\right)$ при различных $t(l+2 r)=\left(t_{1}, \ldots, t_{l+2 r}\right)$ независимы, и следовательно, при любых $h, g, f$

$$
p_{i j}(h, g, f)=\left(\frac{a_{i}}{N}\right)^{r}\left(\frac{a_{j}}{N}\right)^{r}\left(\frac{a_{i}+a_{j}}{N}\right)^{l} .
$$

Отсюда, учитывая, что число слагаемых в сумме по $h, g, f$ равно $(l+2 r) ! /\left((r !)^{2} l !\right)$, получим равенство

$$
D_{1}(l)=\sum_{i \neq j}\left|T_{1}(l+2 r)\right| \frac{(l+2 r) !}{(r !)^{2} l !} \cdots \frac{a_{i}^{r} a_{j}^{r}}{N^{2 r}} \cdots \frac{\left(a_{i}+a_{j}\right)^{l}}{N^{l}} .
$$

Далее нужно воспользоваться формулой (15) с заменой $l$ на $l+2 r$ и провести вычисления, аналогичные вычислениям при выводе формулы (18). После проведения этих вычислений получаем формулу

$$
\begin{aligned}
\sum_{l=0}^{\varphi-1}(-1)^{l} D_{1}(l)=M_{r}^{2} & -\sum_{i=1}^{N} p_{r, i}^{2}-\frac{2 m+1}{N \alpha_{N}}\left((r+1)(r+2) M_{r+2} M_{r}\right. \\
& +2(r+1)^{2} M_{r+1}^{2}-4 r(r+1) M_{r} M_{r+1} \\
& \left.+r(2 r-1) M_{r}^{2}\right)+O\left(N^{\delta}\right) .
\end{aligned}
$$

Сложнее оценивается $D_{2}(l)$. Определим множества

$$
T_{2}^{(u)}(l+2 r, v, n)=\{t(l+2 r): t(l+2 r) \in T(l+2 r)\},
$$

где $t_{v+1}-t_{v}=u, t_{\tilde{v}+1}-t_{\tilde{v}} \geqslant m, \tilde{v} \neq v$.

Вероятности $p_{i j}(h, g, f)$ при $t(l+2 r) \in T_{2}^{(u)}(l+2 r, v, n)$ зависят от размещения $t_{v}$, $t_{v+1}$ по множествам $h, g, f$ :

(1) $t_{v}, t_{v+1} \in h$,

(2) $t_{v}, t_{v+1} \in g$,

(3) $t_{v}, t_{v+1} \in f$,

(4) $t_{v} \in h, t_{v+1} \in g$,

(5) $t_{v} \in g, t_{v+1} \in h$,

(6) $t_{v} \in h, t_{v+1} \in f$,

(7) $t_{v} \in f, t_{v+1} \in h$,

(8) $t_{v} \in f, t_{v+1} \in g$,

(9) $t_{v} \in g, t_{v+1} \in f$. 
Вероятности $p_{i j}(h, g, f)$ в этих вариантах и числа способов разбиения множества $t(2 r+l) \backslash\left\{t_{v}, t_{v+1}\right\}$ на множества $h, g, f$ обозначим соответственно $p_{i j}^{(k)}$ и $S_{k}, k=1, \ldots, 9$.

Приведем числа $S_{k}$ и вероятности $p_{i j}^{(k)}$ :

(1) $p_{i j}^{(1)}=a_{i}^{r-2} b_{i i}(u) a_{j}^{r}\left(a_{i}+a_{j}\right)^{l} / N^{2 r+l}, S_{1}=\frac{(2 r+l-2) !}{r !(r-2) ! l !}$,

(2) $p_{i j}^{(2)}=a_{i}^{r} a_{j}^{r-2} b_{j j}(u)\left(a_{i}+a_{j}\right)^{l} / N^{2 r+l}, S_{2}=S_{1}$,

(3) $p_{i j}^{(3)}=a_{i}^{r} a_{j}^{r}\left(b_{i i}(u)+b_{i j}(u)+b_{j i}(u)+b_{j j}(u)\right)\left(a_{i}+a_{j}\right)^{l-2} / N^{2 r+l}, S_{3}=\frac{(2 r+l-2) !}{(r !)^{2}(l-2) !}$,

(4) $p_{i j}^{(4)}=a_{i}^{r-1} b_{i j}(u) a_{j}^{r-1}\left(a_{i}+a_{j}\right)^{l} / N^{2 r+l}, S_{4}=\frac{(2 r+l-2) !}{((r-1) !)^{2} ! l !}$,

(5) $p_{i j}^{(5)}=a_{j}^{r-1} b_{j i}(u) a_{i}^{r-1}\left(a_{i}+a_{j}\right)^{l} / N^{2 r+l}, S_{5}=S_{4}$,

(6) $p_{i j}^{(6)}=a_{i}^{r-1}\left(b_{i i}(u)+b_{i j}(u)\right) a_{j}^{r}\left(a_{i}+a_{j}\right)^{l-1} / N^{2 r+l}, S_{6}=\frac{(2 r+l-2) !}{(r-1) ! r !(l-1) !}$,

(7) $p_{i j}^{(7)}=a_{i}^{r}\left(b_{j i}(u)+b_{j j}(u)\right) a_{j}^{r-1}\left(a_{i}+a_{j}\right)^{l-1} / N^{2 r+l}, S_{7}=S_{6}$,

(8) $p_{i j}^{(8)}=\left(b_{i i}(u)+b_{j i}(u)\right) a_{i}^{r-1} a_{j}^{r}\left(a_{i}+a_{j}\right)^{l-1} / N^{2 r+l}, S_{8}=S_{6}$,

(9) $p_{i j}^{(9)}=\left(b_{i j}(u)+b_{j j}(u)\right) a_{i}^{r} a_{j}^{r-1}\left(a_{i}+a_{j}\right)^{l-1} / N^{2 r+l}, S_{9}=S_{6}$.

Используя сделанные замечания и равенства (32), получим, что

$$
D_{2}(l)=\sum_{v=1}^{2 r+l-1} \sum_{u=1}^{m} \sum_{t(2 r+l) \in T_{2}(2 r+l)} \sum_{h, g} \sum_{i \neq j} p_{i j}(h, g, f) .
$$

Отсюда следует, что

$$
D_{2}(l)=\sum_{v=1}^{2 r+l-1} \sum_{u=1}^{m}\left|T_{2}^{(u)}(l+2 r, v, n)\right| \sum_{k=1}^{9} \sum_{i \neq j} S_{k} p_{i j}^{(k)} .
$$

Далее нужно воспользоваться равенством (31) из [2]:

$$
\sum_{v=1}^{2 r+l-1}\left|T_{2}^{(u)}(2 r+l, v, n)\right|=\frac{n^{2 r+l-1}}{(2 r+l-2) !}+O\left(\frac{l^{3} n^{2 r+l-2}}{(2 r+l-2) !}\right)
$$

и получить асимптотическую формулу $D_{2}(l)$. Дальнейшие вычисления, связанные с суммированием $D_{2}(l)$ по $l$, аналогичны вычислениям, проведенным в разделе 2 . В схеме 
серий (4), (5)

$$
\begin{aligned}
& \sum_{l=0}^{\varphi-1}(-1)^{l} D_{2}(l)= \frac{2 \alpha_{N}}{N}\left(M_{r} \sum_{i=1}^{N} B_{i i} p_{r, i}+M_{r} \sum_{i=1}^{N} B_{i i} p_{r-2, i}\right. \\
&+\sum_{i, j=1}^{N} B_{i j} p_{r, i} p_{r, j}+\sum_{i, j=1}^{N} B_{i j} p_{r-1, i} p_{r-1, j} \\
&\left.-2 M_{r} \sum_{i=1}^{N} B_{i i} p_{r-1, i}-2 \sum_{i, j=1}^{N} B_{i j} p_{r-1, i} p_{r, j}\right)+O\left(N^{\delta}\right), \\
& \sum_{l=0}^{\varphi-1}(-1)^{l} D_{3}(l)=O\left(N^{\delta}\right), \quad \Delta_{\varphi}=O\left(N^{\delta}\right), \quad 0<\delta<1,
\end{aligned}
$$

где $B_{i j}$ определены равенством (26). Используя (3), (34), (35), получим следующее утверждение.

Теорема 2. При $n, N \rightarrow \infty$ в схеме серий (4), (5)

$$
\begin{aligned}
\mathbf{E} \mu_{r}^{[2]}=M_{r}^{2} & -\sum_{i=1}^{N} p_{r, i}^{2}-\frac{1}{N \alpha_{N}}\left((r+1)(r+2) M_{r+2} M_{r}+(r+1)^{2} M_{r+1}^{2}\right. \\
& \left.-4 r(r+1) M_{r} M_{r+1}+r(2 r-1) M_{r}^{2}\right) \\
& +\frac{2 m}{N \alpha_{N}}\left((r+1)(r+2) M_{r} p_{r+2}+(r+1)^{2} Q_{r+1, r+1}\right. \\
& -2 r(r+1) M_{r} p_{r+1}-2 r(r+1)\left(Q_{r, r+1}+Q_{r+1, r}\right) \\
& \left.+r(r-1) M_{r} p_{r}+r^{2} Q_{r, r}\right)+O\left(N^{\delta}\right),
\end{aligned}
$$

где $0<\delta<1, p_{r, i}, M_{r}, p_{r}, Q_{r_{1}, r_{2}}$ определень равенством (26).

Из теорем 1 и 2 в качестве следствия получаем асимптотическую формулу для $\mathbf{D} \mu_{r}$.

Следствие 1. При $n, N \rightarrow \infty$ в схеме серий (4), (5)

$$
\mathbf{D} \mu_{r}=N \sigma_{N}^{2}(\alpha)+O\left(N^{\delta}\right)
$$

где

$$
\begin{aligned}
\sigma_{N}^{2}(\alpha)=\frac{1}{N} & \left(M_{r}-\sum_{i=1}^{N} p_{r, i}^{2}-\frac{1}{N \alpha_{N}}\left((r+1)^{2}\left(M_{r+1}^{2}-2 m Q_{r+1, r+1}\right)\right.\right. \\
& \left.\left.-2 r(r+1)\left(M_{r} M_{r+1}-m Q_{r, r+1}+Q_{r+1, r}\right)+r^{2}\left(M_{r}^{2}-2 m Q_{r, r}\right)\right)\right),
\end{aligned}
$$

$u 0<\delta<1$.

В случае независимых величин (1), $b_{i j}(u)=a_{i} a_{j}, B_{i j}=m a_{i} a_{j}, p_{r}=Q_{r_{1}, r_{2}}=0$, и полученные асимптотические формулы для $\mathbf{E} \mu_{r}, \mathbf{E} \mu_{r}^{[2]}, \mathbf{D} \mu_{r}$ переходят в известные формулы для этих величин (см. [1]). 


\section{5. Асимптотическая нормальность $\mu_{r}(n, N)$}

Для доказательства асимптотической нормальности $\mu_{r}(n, N)$ воспользуемся вариантом теоремы Янсона [6], приведенным в работе М. И. Тихомировой [3]. Этот вариант незначительно отличается от варианта теоремы Янсона, предложенного В. Г. Михайловым в [7]. Приведем формулировку теоремы 1 из [3] в удобном для использования в рассматриваемой задаче виде.

Будем говорить, что цепочки $\tau\left(t_{i}\right)=\left(t_{i}, t_{i+1}, \ldots, t_{i}+m\right), i=1,2$, пересекаются, если $\tau\left(t_{1}\right) \cap \tau\left(t_{2}\right) \neq \varnothing$. Положим $D_{N}(l) \subset\left\{\gamma=\left(u,\left(t_{1}, \ldots, t_{l}\right)\right): u=l, 1 \leqslant t_{1}<\ldots<t_{l} \leqslant n\right\}$,

$$
\Gamma_{N}\left(L_{N}\right)=\bigcup_{l=1}^{L_{N}} D_{N}(l)
$$

Рассмотрим следующую сумму, определяемую $m$-зависимыми величинами последовательности (1):

$$
S_{N}=\sum_{\gamma \in \Gamma_{N}\left(L_{N}\right)} \eta_{\gamma}
$$

где $\eta_{\gamma}=g_{l}\left(x_{t_{1}}, \ldots, x_{t_{l}}\right),\left|g_{l}\right| \leqslant 1$ при любых значениях $x_{t_{1}}, \ldots, x_{t_{l}}$. Пусть $V-$ произвольное подмножество $\Gamma_{N}\left(L_{N}\right)$. Определим множество $F_{V}$ индексов слагаемых суммы (37), зависящих от слагаемых, соответствующих множеству $V$, полагая

$$
\begin{aligned}
& F_{V}=\left\{\gamma=\left(u,\left(t_{1}, \ldots, t_{u}\right)\right) \text { : найдется } \gamma^{\prime}=\left(v, t_{1}^{\prime}, \ldots, t_{v}^{\prime}\right) \in V\right. \\
& \text { и найдутся такие } \left.1 \leqslant t_{i} \leqslant u, 1 \leqslant t_{j}^{\prime} \leqslant v, \text { что } \tau\left(t_{i}\right) \cap \tau\left(t_{j}^{\prime}\right) \neq \varnothing\right\} .
\end{aligned}
$$

Будем предполагать, что при любом $h$ найдется величина $Q_{h}$ (не зависящая от $V$ ) такая, что при любом $V$ с $|V| \leqslant h$

$H_{V}=\sum_{\gamma \in F_{V}} \mathbf{E}\left(\left|\eta_{\gamma}\right| x_{t} \in G_{V} \mid\right) \leqslant Q_{h}$,

где $G_{V}=\bigcup_{\left(l,\left(t_{1}, \ldots, t_{l}\right)\right) \in V}\left(t_{1}, \ldots, t_{l}\right)$.

Теорема 3. Если при $N \rightarrow \infty$ выполняются условия (4), (5), (39) и при некотором $\beta$, $0<\beta \leqslant 2 / 3$, выполняется условие

$$
\left|\Gamma_{N}\left(L_{N}\right)\right| \rightarrow \infty, \quad \frac{M_{N}^{\beta} Q_{h}^{2-\beta}}{\mathbf{D} S_{n}} \rightarrow 0,
$$

где

$$
M_{N}=\sum_{\gamma \in \Gamma_{N}\left(L_{N}\right)} \mathbf{E}\left|\eta_{\gamma}\right|,
$$

то распределение величины $\left(S_{N}-\mathbf{E} S_{N}\right) / \sqrt{\mathbf{D} S_{N}}$ сходится к стандартному нормальному распределению.

Случайная величина $\mu_{r}(n, N)$ с использованием разбиения (12) и равенства (7) может быть представлена в следующем виде:

$$
\mu_{r}(n, N)=\mu_{r}^{(1)}(N)+\mu_{r}^{(2)}(N)+\mu_{r}^{(3)}(N)+\Delta_{\varphi},
$$


где

$$
\begin{gathered}
\mu_{r}^{(u)}(N)=\sum_{l=r}^{\varphi-1} \sum_{t(l) \in T_{n}(l)} \xi_{l}\left(t_{1}, \ldots, t_{l}\right), \quad u=1,2,3, \\
\xi_{l}\left(t_{1}, \ldots, t_{l}\right)=(-1)^{l+r}\left(\begin{array}{l}
l \\
r
\end{array}\right) \sum_{i=1}^{N} \chi\left(x_{t_{1}}=\ldots=x_{t_{l}}=i\right), \\
\left|\Delta_{\varphi}\right| \leqslant \sum_{u=1}^{3} \bar{\Delta}_{u}, \quad \bar{\Delta}_{u}=\Delta_{u}(\varphi), \quad \Delta_{u}(l)=\sum_{t(l) \in T_{n}(l)}\left|\xi_{l}\left(t_{1}, \ldots, t_{l}\right)\right|, \\
\varphi=\frac{(1+\delta) \ln N}{\ln \ln N}, \quad \delta>0 .
\end{gathered}
$$

Повторяя с несущественными изменения рассуждения, приведенные в [2, 3], можно доказать, что $\left(\mu_{r}(n, N)-\mu_{r}^{(1)}(N)\right) / \sqrt{\mathbf{D} \mu_{r}(n, N)}$ стремится по вероятности к 0 в схеме серий (4), (5) and $\mathbf{D} \mu_{r}^{(1)}(N) / \mathbf{D} \mu_{r}(n, N) \rightarrow 1$, если

$$
\sigma_{N}^{2}(\alpha) \geqslant \varepsilon>0,
$$

где $\sigma_{n}^{2}(\alpha)$ определено в (36). Условие (39) может быть проверено так же, как в [3].

Сформулируем теорему об асимптотической нормальности $\mu_{r}(n, N)$.

Теорема 4. Если в схеме серий (4), (5) выполняется условие (43), то распределение случайной величинь $\left(\mu_{r}(n, N)-\mathbf{E} \mu_{r}(n, N)\right) / \sqrt{\mathbf{D} \mu_{r}(n, N)}$ сходится к стандартному нормальному распределению.

Неравенство (43) выполняется, например, для малых значений $\bar{\alpha}$ при условии (4). Это следует из неравенства

$$
\sigma_{N}^{2}(\alpha)=\alpha^{r} \frac{1}{N} \sum_{i=1}^{N} \frac{a_{i}^{r}}{r !}+O\left(\alpha^{r+1}\right) .
$$

\section{Список литературы}

1. Колчин В. Ф., Севастьянов Б. А., Чистяков В. П., Случайные размещения. Наука, Москва, 1976.

2. Тихомирова М. И., Чистяков В. П., Об асимптотике моментов числа непоявившихся $s$-цепочек. Дискретная математика (1997) 9, №1, 12-29.

3. Тихомирова М. И., Асимптотическая нормальность числа непоявившихся несплошных цепочек исходов независимых испытаний. Дискретная математика (2009) 21, №2, 112-125.

4. Михайлов В. Г., Асимптотическая нормальность в схеме конечно-зависимого размещения частиц по ячейкам. Математический сб. (1982) 119 (161), №4 (12), 509-520.

5. Шойтов А. М., Нормальное приближение в задаче об эквивалентных цепочках. Tpуды по дискретной математике (2007) 10, 326-349.

6. Janson S., Normal convergence by higher semi-invariants with application to sums of dependent random variables and random graphs. Ann. Probab. (1988) 16, №1, 305-312.

7. Михайлов В. Г., Об одной теореме Янсона. Теория вероятностей и ее применения (1991) 36, $168-170$. 